\title{
Liquid Love in Iran: A Mixed Method Approach
}

\author{
Ehsan Shahghasemi \\ PhD Candidate in Communication, Department of Communication, University of Tehran \\ shahghasemi@ut.ac.ir \\ Hosna Masoumi \\ MA graduate in Communication, Department of Communication, University of Tehran \\ Manijeh Akhavan \\ MA graduate in Communication, Department of Communication, University of Tehran \\ Bijan Tafazzoli \\ PhD student in International Relations at Koç University, Turkey \\ btafazzoli14@ku.edu.tr
}

\section{Doi:10.5901/mjss.2015.v6n1s1p138}

\begin{abstract}
This study explores the dimensions of "liquid love" among Iranian Internet users. Over the last decade, the phenomenal growth of the Internet has caused fundamental changes in the Iranian lifestyle. One aspect of the Iranian lifestyle is the strict cultural codes on how males and females can interact. Now, on the Internet, most of this cultural barriers have been removed. It is very important to study how heterosexual relationships are rampant in the Iranian cyberspace, and what are the causes and consequences. Therefore, this study conducts a quantitative study on more than 500 Iranian young Internet users and then analyses 3000 Iranian blog posts which Iranians have written on heterosexual relationships online which Zygmunt Bauman calls it "liquid love." Finally, a comparison of both phases revealed that this kind of relationships are rampant in Iran, and this phenomenon has generally left unpleasant feelings and consequences on the Iranian young Internet users, as discussed in their answers to our questionnaire and their posts on their weblogs.
\end{abstract}

Keywords: Liquid Love; Love Online; Iran; Internet; Chat; Internet Infidelity

\section{Introduction}

Philosophical perspective on the media holds that the nature of technology has immense effects on the way we see the world. Some, like Canadian scholar Harold Innis believe that changes in communication technologies pave the way for development of a new civilization. After more than two decades of fast development of the Internet, there has been much debate on its impact on the human society.

Over the last decade the growth of Internet in Iran has marked one of the fastest in the world. Based on formal stats, in June 2014, about half of the more than 75 million Iranian population were Internet users, which is very high in a region in which the rate of illiteracy is still high. Based on the reports of Global Campaign for Education, there are 97 million illiterate citizens in the Arab world and the rate of illiteracy is 50 percent for Pakistan and 72 percent for Afghanistan. Does this stats mean Iran is pioneer in utilizing ICTs for development? The recent studies do not support this hypothesis. The research have shown that a considerable amount of internet use in Iran is allocated to heterosexual relationships (Hanassab, 1996; Manteghi, 2004; Pak Seresht, 2006; Iman \& Boostani, 2010; Ahmadi, et al., 2012; Javadi Yeganeh, et al., 2012; Del Avar \& Moghadas, 2012; Rahmani, et al., 2013).

But, this is a new situation. For thousands of years, heterosexual relationships were more or less dominated by face-to-face communication and interaction. Also, these relationships occurred in a social setting in which both parties assumed certain responsibilities. Failing to keep these responsibilities would damage social reputation of people and even could culminate to punishments. Online relationships have removed these barriers to a high level and now a considerable number of citizens are looking for online love (Craft, 2012). The -perceived- anonymity and lack of social 
obstacles provides the possibility of cutting bonds without suffering consequences. In his marvelous book Liquid Love: On the Frailty of Human Bonds, Zygmunt Bauman (2003, p. XII) quotes a 28 years old man who when asked what was the advantage of cyber relations in comparison to real ones, said "you can always press delete."

This study aims to answer fundamental questions about "liquid love" in Iran: how rampant it is? What do young users believe about it? What are its concequences on the real life of the users? And how it affects factors like worldview, sense of community, sociability, etc. There have been some studies with the same approach in other countries, but this is the first study on this subject with this theoretical framework in Iran.

\section{Conceptual Framework}

Although online relationships could be studied from dozens of approaches from different disciplines, the psychological, social and technological traits of these relationships convinced us to combine two theoretical perspectives to study them. The first viewpoint was the notion of "liquid love" by Zygmunt Bauman. Bauman wrote Liquid Love: On the Frailty of Human Bonds in 2003 to criticize the roots and nature of online relations in what he called "liquid modernity." Bauman was previously understood as a postmodern critic, since he thought no single viewpoint can capture enough information about a single phenomenon. But, he gradually retreated from postmodernism and chose to use phrase "liquid" to procedures in our new world. He thinks our lives have changed in a way to become like a liquid that takes the shape of its container. The dimensions of this change is so immense that affects all aspects of our lives, including our intimate relations. Therefore, as we are now used to change our laptop, cellphone, car and furniture every year or so, we may be tempted to do the same to our emotions. In a world that nothing lasts for long, the intimate, enduring and genuine love has no place.

Therefore, this study would study liquid love among the Iranian users to see how this phenomenon is rampant in Iran and in what quality and quantity. But, we will study this notion in a dual-spacized paradigm. This paradigm was first introduced by Ameli (2003) to show how rapid growth of new communication technologies have had produced a twin world of virtual and real spaces which are paradoxically detached and intertwined with each other. Hence, what affects our life in the virtual world will have repercussions in the real world and vice versa. This paradigm, therefore, will predict how liquid love, which may be perceived as nothing other than a dating activity in nowhere, will have important consequences in the real life of the users. Since 2003, Ameli has applied his theories in many fields including civilization, e-government, virtual city, simulation, critical studies, intercultural communication, etc.

\section{Methodology}

This is a mixed method research which was carried out in two consequent phases. In phase one we ran a survey. We designed a questionnaire with close ended questions based on Likert scale and ran a pretest among students at the University of Tehran. A number of 69 students answered the print questionnaire and a factor analysis test in SPSS software shown that 4 questions should be excluded because of inconsistency. The new questionnaire with replaced questions were then made online and a private corporation send thousands of emails to Iranian Internet users, inviting them to answer the questionnaire. Within two weeks, about 600 users answered the questionnaire but as the range of age in this research was 16-32, participants out of this range were excluded.

After analyzing the results, the general conclusions were drawn and a background was made available to run the second phase of the study which was qualitative analysis on how the young Iranian users saw heterosexual relationships on the Internet. In order to make sure that comments and posts analyzed in this phase were personal, and not news or commentary by professional journalists or officials, we only included material from blog providers like Blogfa, Persianblog, blogsky etc. Qualitative analysis of the weblogs was carried out to reveal categories and then the process stopped only when saturation was obtained and no further categories were produced. This, included material from almost 3000 weblogs. At the end, we compared the results of both phases to see if there was any relationships between the two.

\section{Findings}

\subsection{Quantitative Findings}

493 out of 530 participants were demographically fit into this study, among them about 62 percent were male. About 38 percent of them held a BA degree and another 37 percent said they got either a MA or MS degree. This profile shows that the participants in this study were generally well educated. Factor analysis was run again (as we mentioned, a pre-test 
study had been carried out earlier) and all clusters of questions proved to pass Alpha Cronbach test.

Analyzing the findings of this section showed that about 64 percent of 493 participants are agree or strongly agree with relationships before marriage. But, 53 percent of participants said they dislike or strongly dislike having relationships online. Also, 87 percent of the participants expressed their cosmopolitan intentions which shows a majority of the participants in this survey are not to be considered right wing or buy ideas against others.

Crosstabulations offer more interesting data. With Pearson correlation coefficient of -0.008 , there is no significant relationship between age and intention to have online relations. Also, no significant relationship was found between gender and intention to have online relations. As regard to relationship between level of education and intention to have online relations, LSD test showed that there is a reverse correlation between level of education and intention to have online relations which shows that people with higher levels of education are less apt to make virtual relations with other sex.

Our findings revealed that those who have more positive views regarding relationships before marriage are more likely to be positive about making online relations $(R=0.0335)$. Also, we found those who are more controlled by their families and social circle and are not to have relationships outside or before marriage, are more likely to be positive about having online relations $(\mathrm{R}=0159)$.

One important finding in our study was to learn how online relations will affect individual's real relationship. There is a direct correlation between reclusion and establishing virtual relations with the opposite sex $(R=0.159)$. Also, we found there is weak, but significant direct correlation between having cosmopolitan ideas and being positive about online heterosexual relationships $(\mathrm{R}=0.383)$.

\subsubsection{Path Analysis}

After doing the preliminary analysis, a path analysis will help us figure out how our variables relate to each other and can predict each other's change. Here, we first create a causal one way model and then we calculate the correspondent coefficient. At the end, the final sum of beta coefficients which are applied to end independent variable, will be a total effect. In path analysis we try to increase $R^{2}$ and decrease $K^{2}$. Therefore, $R^{2}+K^{2}$ will be equal to 1 .

To draw a diagram for path analysis, first we incorporated only those variables which according to our findings have proved to have significant relation to dependent variable. We will put them into regression system to measure their effect on dependent variable. In the next steps, we put each of these variables will be put in the regression system as a dependent variable and we will study the effects of other variables on it.

Here, our variables are: Being positive on having relationships before marriage; Intention to have online relations; More control by family or social circle not to have relationships outside or before marriage; and, intention towards other cultures. After putting our variables in regression analysis, we have found the following diagram:

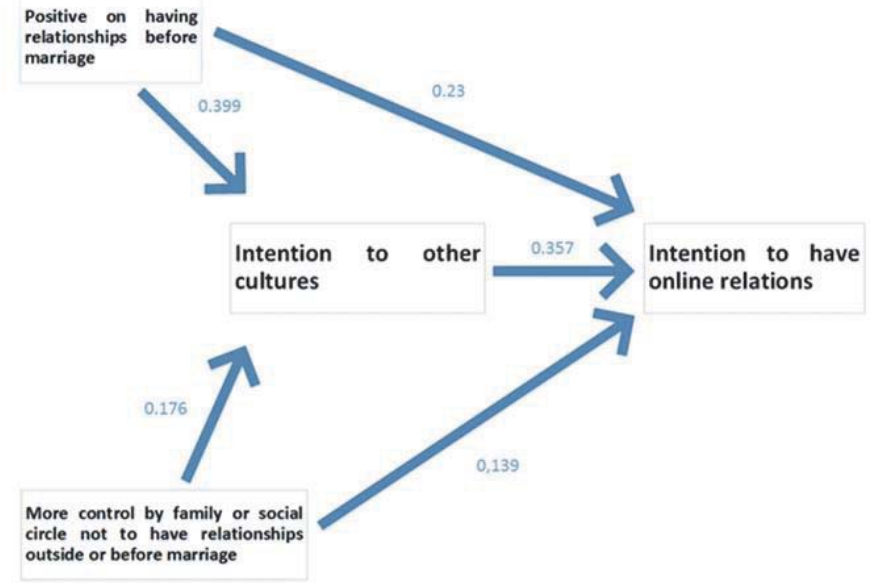

\subsection{Qualitative Findings}

After evaluating the results of the first phase of this study, we managed to design our qualitative investigation. As we said earlier in this paper, Iranian cyberspace is vivid and Iranian people have proven to be apt to publish their ideas on the 
Internet. Blogging is one of the ways Iranians have taken to publish their opinions (see for example (Drezner \& Farrell, 2004) and (Doostdar, 2004)). Also, Iranian culture is categorized among complex cultures in which realities are sometimes expressed with a high degree of caution and on many issues, including personal matters, people are highly conscious not to be open among those whom they know. Perceived anonymity in the blogsphere and de-personalized nature of blogging make Iranians less prudent in writing on online relations and this will provide plenty of reliable information for researchers.

In this study, as we said in the methodology section, about 3000 posts on the Iranian Persian language weblogs were collected. Each post in each weblog was taken as one unit of analysis and in any weblog, only one post could be included in the study. The whole gathered material was put in a same document. We read through the material three times and codified categories and subcategories. Then we read the material again and put any post in one of these categories. We found three main categories consisting of negative, positive, and mixed comments about liquid love in Iran.

\title{
4.2.1 Negative Views on Liquid Love
}

About 62 percent of the posts discussing the matter of online heterosexual relations were more intended to criticize these relationships. The hollow nature of these relationships, deception, depression, reclusion and failure to fulfil personal duties due to excessive time allocated to wander in chatrooms, were among their reasons to criticize liquid love. Our findings showed that most of these bloggers drew on their own experiences when they were criticizing online relationships.

As we analyzed this category, we decided that the material could be divided to two subcategories: negative views on consequences of online relations, and negative views on seductive nature of online relations.

\subsubsection{Negative Views on Negative Consequences of Liquid Love}

These comments and posts varied on many aspects. Iranian bloggers mentioned a range of negative consequences from depression and paranoia, to death threat and abduction. It seems the Iranian society is at the same time well aware of unwanted concequences of cyber relations and loves it. Examples in this subcategory are:

\begin{abstract}
Neshmil
Iranian boys are more apt to chat online and this will culminate in many consequences and mental disorder!!! (What a pity). . . I have myself . . . suffered the consequences of this more sweet than honey joy!!! And I have suffered badly!!! And now that I am writing this, I regret it!!!

Mihah lav

You only need to create an ID with a female name and enter chatroom. . There are married people who are looking for mates, just like single people. Married men are more frequently seen in chatrooms as compared to married women. . . . When questioned why they do this, some of them say they do not love their wife or husband.

Asemooni

You can see that smileys which are sent by the most dignified "chatters" are sometimes very abnormal. . the charm of false personalities and the possibility to construct faked personalities has provided a way for boys and girls of any age groups to befriend. Unfortunately, these relationships have in many cases culminated in unfitted marriages which sooner or later will eventually end up in divorce . . . the research have shown that 90 percent of girls who approach virtual friendships are looking for satisfaction of their minimum needs which means the need to speak to other sex forces them into a self-deception that this man is right and perhaps he is different from others.
\end{abstract}

\subsubsection{Negative Views on Seductive Nature of Liquid Love}

One of the issues that Bauman raised about online relations, is deception and its changed nature in virtual relations. As cyberspace becomes a haven for deceptive behavior (Galanxhi \& Fui-Hoon Nah, 2007), the stories of deception online is now something usual in the media. Iranian bloggers have taken up the issue and scrutinized it and its effects.

\section{Zahra Shabani}

I feel pity for those parents who when their sons and daughters lock themselves in the room to chat with their friends in chatroom, feel good that their kids are hardly working to study and say my kid has worked so hard that he or she is about to collapse! ... a coffee net owner had said in interview that he knew a 60 year old man in his coffee net had asked him to make an ID of a single 24 years old boy! 


\title{
Sarghad
}

While one cannot even trust his old friends in the real world, how can he or she trust somebody in the cyberspace whose identity is neither determined, nor verifiable? Even if he or she sends a photo, the possibility of deception will be still in place. I can say I am handsome, good looking, well educated, wealthy, and fluent in several languages; who can prove I am not?

Sokhan-e Ashena

One should generally note that one important principle in the cyberspace is non-trust and hence, one should not direct his or her relationships to real ones. It is especially important for women.

Ezlove

Some women who work with websites and weblogs in the cyberspace must be cautious. . . some women are deceived by physical attractiveness ... and in cyberspace there is a possibility that because of their high level of imagination, people become seduced.

Sahba

In cyberspace, many people can lie, intimidate, deceive or steal other's identities without fear. Even they can make different separate IDs in websites like Facebook to have several online lives. . the research have shown that one third of individuals in the friend finding websites have faked identities and 45 percent of them lie about their personal data.

\subsubsection{Mixed Attitudes towards Liquid Love}

About 15 percent of the comments in this study fell into this category. In this category, Iranian bloggers did not express pure positive or negative comments about liquid love. Philosophically, most of the comments in this category had an instrumental view towards the cyberspace which means they thought the cyberspace is not in itself something bad or good but, it is the way we use it that makes it good or evil.

\begin{abstract}
Sevom Computer Moshfegh Khor
In these years many became happy and some even married. Some died and some. . . To be honest, most of the guys in chat are very nice. As a personal experience, every technical problems regarding computer which I had asked, all of them, good and evil, took time and answered my questions. . . . I don't say chatting is good or bad. It has many good things and many...

Sara va Adel

Many girls have found chatrooms a safe place for establishing relationships with the other sex because no harm will be made to them. They can easily chat, without being stared at, or somebody hear their voices or even know their names. In this way, they will get answers to their myriad questions about the opposite sex and at the same time they will stay safe from possible challenges. But, there has been instances in which befriending through chatrooms have put girls in trouble. . . why most of us still cannot have an optimum utilization from it?

Habil

This is a phenomenon which has become a part of our long lonely days . . In the categorized and cast society of Iran in which everyone's place is determined by his appearance, and that who is rich is necessarily known noble, and fondness of appearance has become a culture, every mask which conceals deficits and shortcomings must be embraced. In chat you become what you want. You can make your aspirations true ... this is only a scape from ourselves.
\end{abstract}

\subsubsection{Positive Comments about Liquid Love}

This group of posts constitute 23 percent of attitudes expressed by Iranian bloggers. It seems that those Iranian youth who have positive perceptions of liquid love more or less see this phenomenon as a personal and appealing experience of love.

\section{Amirbarf}

At a night without you, I become online, and I become all eyes, searching for you. The thrill of meeting you overwhelmed my existence. . . I became the same mad man again. . . in the middle of the screen, your memoirs shined, and the sound of ding filled the space...

Nima

I found many good friends in chatrooms; wise and knowledgeable friends. I looked for someone who could understand me and listen to me and we can help each other. Some nights ago, I was chatting to my friend (Behnoush) whom I really love...

Mohammad

Fateme is the name of a girl with whom I fell in love. I got to know her in a long day at the end of the autumn in a coffee net. I spotted her ID from her screen and started to chat with her. . . she is know my mistress.

Kamran va Hooman 
My mama doesn't let me come into chatroom but now I have seduced her and came over. It is now my turn.

Shahbaz

In my opinion ..., friendship and love in the virtual space can support love and friendship in the real world. I have been honored and I am honored to meet in person with some people with whom I become acquainted in chatrooms and I know even like them more.

Nashenas

I am a 21 years old boy and I am a college student. Some years ago I got in contact with a girl through telephone and email. She is my age and lives in another city. For one year, we communicated over phone and exchanged photos, love letters and gifts ... I think she is my lost half ... we understand each other. We did not choose each other based on appearance. We loved each other through our attitudes and ways of thinking.

\section{Discussion and Conclusion}

Over the last two decades the introduction of new communication technologies have changed our communication landscape dramatically. We are no longer solely members of our community or local society. One result of this new situation, is the formation and spread of what Zygmunt Bauman called "Liquid Love."

As liquid love's first feature is its possibility to escape human bonds, we can predict that in societies that there is higher level of control and surveillance, the possibility of establishing liquid love through cyberspace should be theoretically higher. As our study shows, Iranians as people who live in a traditional society with traditional bonds are eager to experience and even addict to love online. Also, as the zeal to have heterosexual relations increases, the amount of time spending on liquid love through cyberspace increases.

One other important finding of this study is that both qualitative and quantitative parts are supporting each other in explaining what negative consequences liquid love may impose on the Iranian users. Particularly, we see in both parts how users and particularly bloggers expressed the problems liquid love create to put the users in despair, depression, loneliness, anxiety, and failure to fulfil their daily routines and duties. One other intriguing findings in this study is that, a majority of those 63 percent of the Iranian bloggers who have expressed pure negative comments about love online, have shown in their comments that they have had experienced it themselves and they had used to have positive comments about liquid love. This means among those bloggers who have expressed positive comments about the liquid love, there are a considerable number who will turn to become pessimists about liquid love.

We should not forget that despite the many negative effects and consequences that liquid love have for the Iranian users, there are positive consequences which should not be overlooked. People will find information about other possibly different- people and hence they learn to manage differences or in the better word, bridge the differences. Also, people may develop computer or network skills which help them to find a job in the future or at least act more effectively in the cyberspace. One other possibility is that people get shocked by being harmed in the cyberspace but remain to some extent safe in the real world -though our findings show that people will never get rid of the problems they faced in cyberspace, in the real world- and act better in the real world.

But, in general, we like Zygmunt Bauman think these relationships are generally against the human nature and emotional needs and in most cases will culminate in depression, pessimism, despair, and misbehavior.

\section{Acknowledgment}

Authors like to acknowledge the invaluable help of Professor Saied Reza Ameli from the University of Tehran. We also like to thanks professor Zygmunt Bauman for his wonderful recommendations and support.

\section{References}

Ahmadi, K., Akhavi, Z. \& Abdolmaleki, H., 2012. Naghshe vizhegihaye shakhsiati dar doostihaye Interneti (chat). Journal of Research in Phsychological Helath, 6(2), pp. 31-39.

Ameli , S. R., 2003. Do jahani shodanha va jame'eye jahanie ezterab. Social Sciences Letter, Volume 21, pp. 143-174.

Bauman, Z., 2003. Liquid Love: On the frailty of human bonds. Cambridge: Polity Press.

Craft, A. J., 2012. Love 2.0: A quantitative exploration of sex and relationships in the virtual world. Archives of Sexual Behavior, 41(4), pp. 939-947.

Del Avar, M. S. \& Moghadas, A. A., 2012. Rabeteye estefade az fanavarihaye ertebatati va etela'ati bar ertebat ba jense mokhalef. Bianual Journal of Social Sciences, 2(2), pp. 1-24.

Doostdar, A., 2004. "The Vulgar Spirit of Blogging": On language, culture, and power in Persian weblogestan. American Anthropologist, 106(4), p. 651-662. 
Drezner , D. W. \& Farrell, H., 2004. Web of influence. Foreign Policy, Volume 145, pp. 32-40.

Galanxhi, H. \& Fui-Hoon Nah, F., 2007. Deception in cyberspace: A comparison of text-only vs. avatar-supported medium. International Journal of Human-Computer Studies, 65(9), p. 770-783.

Hanassab, S., 1996. Intramarriage and intermarriage: Young Iranians in Los Angeles. International Journal of Intercultural Relations, 22(4), p. 395-408.

Iman, M. T. \& Boostani, D., 2010. Tahlile keifie peivand faraghat va hoviat dar miane danesh amoozane dabirestanie Shiraz. Bianual Journal of Sociel Sciences at Faculty of Humanities and Literature, 7(2), pp. 1-35.

Javadi Yeganeh, M. R., Kousari, M. \& Khairkhah, T., 2012. Telefone hamrah va karkardhaye an baraye karbarane Irani ba takid bar tafavothaie jensiati. Social Research and Investigation in Iran, 1(2), pp. 23-54.

Manteghi, M., 2004. Raftar shenasie javan dar daheye sevom va chaharome enghelabe eslami. Tehran: Pajooheshgahe Oloome Ensani.

Pak Seresht, S., 2006. Masrafe Internet dar sabkhaye faraghatie javanane Tehrani. Cultural Stuies and Communication Quarterly, 2(5), pp. 137-158.

Rahmani, M. A., Yeganeh, T. \& Morad Hosseini, M., 2013. Barresie rabete beine ehsase tanhaei va ba etiad be Internet va rabete ba jense mokhalef dar nojavanan. Tabriz, Tabriz University of Medical Sciences.

Shahghasemi, E., Tafazzoli, B. (2013). Cross Cultural Schemata: How Do Iranian Bloggers View the American People? Online International Journal of Arts and Humanities, 2(7), 182-188. 